\title{
Product Integration Over Infinite Intervals I. Rules Based on the Zeros of Hermite Polynomials
}

\author{
By William E. Smith, Ian H. Sloan and Alex H. Opie
}

\begin{abstract}
The paper discusses both theoretical properties and practical implementation of product integration rules of the form

$$
\int_{-\infty}^{\infty} k(x) f(x) d x \approx \sum_{i=1}^{n} w_{n i} f\left(x_{n i}\right),
$$

where $f$ is continuous, $k$ is absolutely integrable, the nodes $\left\{x_{n i}\right\}$ are roots of the Hermite polynomials $H_{n}(x)$, and the weights $\left\{w_{n i}\right\}$ are chosen so that the rule is exact if $f$ is any polynomial of degree $<n$. Convergence of the rule to the exact integral as $n \rightarrow \infty$ is proved for a wide class of functions $f$ and $k$ (including singular or oscillatory functions $k$ ), and rates of convergence are estimated. The rules are shown to have the property of asymptotic positivity, and as a consequence exhibit good numerical stability. Numerical calculations for some practical cases are presented, which show the method to be computationally effective for integrands (including highly oscillatory ones) that decay suitably at infinity. Applications of the method to integration over $[0, \infty)$ are also discussed.
\end{abstract}

1. Introduction. In this paper we propose a product-integration method, in the sense of [2], [5], [6], [18]-[22], [25], for evaluating an integral of the form

$$
I(k, f)=\int_{-\infty}^{\infty} k(x) f(x) d x
$$

where $f$ is a smooth function and $k$ is a Lebesgue integrable function. Additional conditions on $k$ and $f$ will be imposed later.

The method will also handle integrals over semi-infinite intervals of the analogous form

$$
I_{+}(k, f)=\int_{0}^{\infty} k(x) f(x) d x,
$$

since there exist simple transformations that convert integrals of this form into the form (1.1) - see Section 6 for details.

The product-integration method is based on approximating the smooth function $f$ by a polynomial. More precisely, the integral $I(k, f)$ is approximated by

$$
I_{n}(k, f)=\int_{-\infty}^{\infty} k(x) L_{n}^{f}(x) d x,
$$

Received March 18, 1982.

1980 Mathematics Subject Classification. Primary 65D30, 4lA55; Secondary 41A05, 41A10, 65D05. $33 \mathrm{~A} 65$

Key words and phrases. Numerical integration, infinite interval, product integration, interpolation, Hermite polynomials. 
where $L_{n}^{f}$ is the unique polynomial of degree $\leqslant n-1$ that coincides with $f$ at $n$ preassigned points $x_{n 1}, \ldots, x_{n n}$. It is well known, at least for a finite interval, that polynomial interpolation is an uncertain matter unless the interpolation points $x_{n i}$ are well chosen. In the present work we take the $n$ points to be the zeros of the $n$th degree Hermite polynomial $H_{n}(x)$. With that choice of points we shall show that a very satisfactory convergence theory results and also that very satisfactory rates of convergence can be obtained for suitable choices of $k$ and $f$.

Since the fundamental notion is that of approximation by a polynomial, we might expect that for rapid convergence $f$ needs to be smooth, and not grow too rapidly at infinity; and that $k$ should decay at infinity, in order to suppress the growth of the approximating polynomial. More precise growth conditions will be stated later.

A more practical expression for $I_{n}(k, f)$ may be obtained by substituting into (1.2) the Lagrange form of the interpolating polynomial $L_{n}^{f}$, to obtain an expression of the form

$$
I_{n}(k, f)=\sum_{i=1}^{n} w_{n i}(k) f\left(x_{n i}\right) .
$$

An explicit expression for the weights $w_{n i}(k)$ is given in Section 5 . The resulting quadrature rule is clearly exact if $f$ is any polynomial of degree $<n$, and in fact that property gives an alternative characterization of the product-integration rule.

If $k(x)=e^{-x^{2}}$, the rule reduces to the classical Gauss-Hermite quadrature rule. In this case the rule is exact if $f$ is any polynomial of degree $<2 n$, and the weights $w_{n i}$ are all positive. In other cases the rule is not a Gaussian rule, because the points $x_{n i}$ remain fixed as $k$ varies. Note that the rule, unlike a Gaussian rule, places no restrictions on the sign of $k(x)$, and in fact some of the most interesting applications are those in which $k$ is highly oscillatory. (See Section 7 for numerical examples.) In general the weights, unlike Gaussian weights, are not necessarily positive. Nevertheless they turn out to be extremely well behaved: if $k$ is positive over a subinterval and satisfies a specified integrability condition, then the weights corresponding to that subinterval are 'asymptotically positive' in the sense in [21]. The precise condition is stated in Section 2. Also stated there are general theorems on the convergence and rate of convergence of $I_{n}(k, f)$ to $I(k, f)$.

Gabutti [9] and Patterson [16] have evaluated certain infinite oscillatory integrals by an extension of the method proposed by Bakhvalov and Vasil'eva [2]. In this method the function $f$ is first approximated by the partial sum of an appropriate orthogonal polynomial series, and then the coefficients in that sum are evaluated approximately by Gaussian quadrature. As was recognized by Patterson [16], this method can be reinterpreted as an interpolatory product integration method, the points $x_{n i}$ being the nodes of the Gaussian quadrature. However, this approach does not appear to lead readily to full theories of convergence, rates of convergence or numerical stability. These are obtained in the present work by extending the methods used in some recent studies of product integration on the finite interval [19], [21], [22].

A quite different method for handling oscillatory integrals over semi-infinite intervals is the acceleration method, which has been reviewed and extended by Blakemore, Evans and Hyslop [3]. That method is based on the use of accurate 
quadrature rules to integrate between successive zeros, combined with powerful acceleration techniques to speed the convergence as the upper limit is taken to infinity.

Finally, we mention that there exists a quite different product-integration approach from the one studied in this paper, namely to transform the integral to one over a finite interval, and then to apply a polynomial product-integration rule to the finite interval. Transformations of this kind are discussed in [5]. The rule so obtained can be viewed as a product-integration rule for the original interval, based not on polynomials but instead on the functions obtained from the polynomials by the transformation from the finite to the infinite interval. A method of this kind has been used very successfully by Lehman, Parke and Maximon [12] for a class of semi-infinite intervals involving spherical Bessel functions; their method for the finite interval is that of Sloan and Smith [19], [20]. The success of such an approach rests, of course, on the transformed function $f$ being a smooth function over the finite interval, and hence easily approximated by a polynomial. If that is the case, then this approach can be expected to work very well; and for cases in which $k(x)$ does not decay rapidly at infinity it is likely to be preferable to the method studied in this paper. But if $k(x)$ does decay rapidly, then the present approach of using polynomials over the infinite interval seems more straightforward.

The principal theoretical results of the paper are stated and discussed in Section 2 and proved in succeeding sections. The practical construction of the rule for various classes of function $k(x)$ is taken up in Section 5. Integrals on $[0, \infty)$ are discussed in Section 6. Some numerical results are given in Section 7.

2. Principal Theoretical Results. The first result is the main theoretical result of the paper. It parallels known results for finite intervals [21], and is proved in Section 3.

THEOREM 1. Let $k$ satisfy

$$
\int_{-\infty}^{\infty}\left|k(x) e^{x^{2} / 2}\right| p d x<\infty
$$

for some $p>1$, and let $f$ be a function which is Riemann integrable on every finite interval, and which satisfies

$$
|f(x)| \leqslant c \frac{e^{x^{2} / 2}}{\left(1+x^{2}\right)^{1 / 2}}
$$

for some constant c. Then $k f \in L_{1}(-\infty, \infty)$,

$$
\lim _{n \rightarrow \infty} \sum_{i=1}^{n} w_{n i}(k) f\left(x_{n i}\right)=\int_{-\infty}^{\infty} k(x) f(x) d x,
$$

and

$$
\lim _{n \rightarrow \infty} \sum_{i=1}^{n}\left|w_{n i}(k)\right| f\left(x_{n i}\right)=\int_{-\infty}^{\infty}|k(x)| f(x) d x
$$

Remark. The theorem asserts not only the convergence of the quadrature rule itself, under suitable conditions on $k$ and $f$, but also the convergence of a companion rule obtained by replacing all weights by their absolute values. The main significance 
of the companion rule, discussed for the case of a finite interval in [21], is that it ensures numerical stability. Note that if $f$ is the characteristic function of an interval $[a, b]$, then, for all $k$ satisfying the conditions of the theorem, we have from (2.4)

$$
\lim _{n \rightarrow \infty} \sum_{x_{n i} \in[a, b]}\left|w_{n i}(k)\right|=\int_{a}^{h}|k(x)| d x,
$$

and also from (2.3) the same result with the absolute value signs removed. These two conditions, holding as they do for an arbitrary interval, force the weights to reflect very accurately the local behavior of $k(x)$. For example, if $k$ is nonnegative over $[a, b]$, then we obtain from the two conditions

$$
\lim _{n \rightarrow \infty} \sum_{x_{n i} \in[a, b]}\left[\left|w_{n i}(k)\right|-w_{n i}(k)\right]=0 .
$$

from which it follows that the sum of all negative weights corresponding to the interval $[a, b]$ can be made arbitrarily small by taking $n$ sufficiently large-in other words, the weights corresponding to the interval $[a, b]$ are 'asymptotically positive'.

The next result gives an error bound for the quadrature rule (1.3) and generalizes an analogous result for the finite interval [19, Theorem 4]. The bound is computable, at least in principle.

The bound depends, as we would expect, on how well $f$ can be approximated by a polynomial of degree $<n$. However, on an infinite interval there are many different ways in which the quality of the approximation might reasonably be measured. We shall use weighted uniform approximation with respect to a function $g$, where $g$ is positive and continuous on the real line and has the property that $p(x) / g(x) \rightarrow 0$ as $|x| \rightarrow \infty$ for every polynomial $p$, but is otherwise unspecified. Thus we define the error of approximation by

$$
E_{n}(f ; g)=\inf _{p \in P_{n}}\|(f-p) / g\|_{\infty},
$$

where $P_{n}$ is the set of polynomials of degree $\leqslant n$, and the norm is the supremum norm,

$$
\|u\|_{\infty}=\sup _{x \in \mathbf{R}}|u(x)|
$$

THEOREM 2. Let $f$ be a continuous function satisfying

$$
|f(x)| \leqslant c g(x)
$$

where $g$ has the properties stated above and $c$ is a constant, and let $k$ satisfy

$$
\int_{-\infty}^{\infty}|k(x)| g(x) d x<\infty .
$$

Then

$$
\left|I(k, f)-I_{n}(k, f)\right| \leqslant\left[\int_{-\infty}^{\infty}|k(x)| g(x) d x+\sum_{i=1}^{n}\left|w_{n i}(k)\right| g\left(x_{n i}\right)\right] E_{n-1}(f ; g) .
$$

The proof, which is elementary, is given in Section 4.

Theorem 2 can be applied even if $k(x)$ does not satisfy the conditions in Theorem 1. In that case the sum $\Sigma\left|w_{n i}(k)\right| g\left(x_{n i}\right)$ may grow without bound as $n$ increases, but 
a satisfactory rate of convergence might still be achieved if $E_{n-1}(f ; g)$ approaches zero fast enough. (However, the numerical stability will be poor if the sum becomes too large.)

In the present work we are most interested in the case in which $k(x)$ satisfies the conditions of Theorem 1 . For this case we set $g=h$, where

$$
h(x)=\frac{e^{x^{2} / 2}}{\left(1+x^{2}\right)^{1 / 2}}
$$

and deduce the following corollary.

COROLlaRY 1. If $f$ is a continuous function satisfying $|f(x)| \leqslant \operatorname{ch}(x)$, and if $k$ satisfies the condition in Theorem 1 , then

$$
\left|I(k, f)-I_{n}(k, f)\right| \leqslant\left[2 \int_{-\infty}^{\infty}|k(x)| h(x) d x+\varepsilon_{n}(k)\right] E_{n-1}(f ; h),
$$

where $\varepsilon_{n}(k) \rightarrow 0$ as $n \rightarrow \infty$.

The quantity $\varepsilon_{n}(k)$ in the corollary is given by

$$
\varepsilon_{n}(k)=\sum_{i=1}^{n}\left|w_{n i}(k)\right| h\left(x_{n i}\right)-\int_{-\infty}^{\infty}|k(x)| h(x) d x .
$$

Its convergence to zero follows from Theorem 1 with $f$ set equal to $h$.

Asymptotically, the error bound given by Corollary 1 is proportional to $E_{n-1}(f ; h)$. Freud [7, Theorem 3.9] has given bounds on this quantity in terms of a generalized modulus of continuity of $f$. We refer to Freud's paper for details, and content ourselves here with a simply-stated weaker result that follows from Freud's result: if $f$ is $r$ times continuously differentiable, and if

$$
\left|f^{(r)}(x)\right| \leqslant \operatorname{ch}(x)
$$

where $c$ is a constant, then

$$
E_{n}(f ; h) \leqslant C n^{-r / 2},
$$

where $C$ is a constant for given $r$ and $f$.

In rough terms there are two factors that influence the rate at which $E_{n}(f ; h)$ approaches zero, namely the smoothness of $f$ and its rate of growth; the most favorable situation being that of high smoothness and slow growth. If, for example, $f(x)=e^{x^{2} / 2}\left(1+x^{2}\right)^{-3 / 2}$, then the condition (2.6) is satisfied with $r=2$, and it then follows from (2.7) that $E_{n}(f ; h)=O(1 / n)$. As a second example, if $f(x)=e^{x^{2} / 4}$, then (2.6) is satisfied with $r$ taken to be any natural number. Finally, if $f(x)=h(x)$ $=e^{x^{2} / 2}\left(1+x^{2}\right)^{-1 / 2}$, so that $f$ has the maximum rate of growth allowed by Theorem 1 , then Corollary 1 gives no information at all about the rate of convergence, since it can very easily be seen that $E_{n}(h ; h) \geqslant 1$. Even in this case we are assured by Theorem 1 that the quadrature rule converges to the exact result, but the rate of convergence may be very slow. 
3. Proof of Theorem 1. If $k$ and $f$ satisfy the stated conditions, then it follows from the Hölder inequality that $k f \in L_{1}(-\infty, \infty)$, since

$$
\begin{gathered}
\int_{-\infty}^{\infty}|k(x) f(x)| d x=\int_{-\infty}^{\infty}\left|k(x) e^{x^{2} / 2}\right|\left|f(x) e^{-x^{2} / 2}\right| d x \\
\leqslant\left[\int_{-\infty}^{\infty}\left|k(x) e^{x^{2} / 2}\right|^{p} d x\right]^{1 / p}\left[\int_{-\infty}^{\infty}\left|f(x) e^{-x^{2} / 2}\right|^{q} d x\right]^{1 / q} \\
\leqslant c\left[\int_{-\infty}^{\infty}\left|k(x) e^{x^{2} / 2}\right|^{p} d x\right]^{1 / p}\left[\int_{-\infty}^{\infty}\left(1+x^{2}\right)^{-q / 2} d x\right]^{1 / q},
\end{gathered}
$$

where $1 / q=1-1 / p$. The last integral is finite because $q>1$, and the first is finite by assumption.

The proof of the main results is based on the following mean convergence result of Nevai [14, Theorem 1] for polynomial interpolation at the zeros of $H_{n}(x)$.

THEOREM. Let $f \in C(\mathbf{R})$ and be such that

$$
\lim _{|x| \rightarrow \infty} f(x) x e^{-x^{2} / 2}=0 .
$$

Then, for every number $q$ satisfying $1<q<\infty$, we have

$$
\lim _{n \rightarrow \infty} \int_{-\infty}^{\infty}\left|L_{n}^{f}(x)-f(x)\right|^{q} e^{-q x^{2} / 2} d x=0 .
$$

The first convergence result in Theorem 1 can be obtained for a more restricted class of function $f$ by a simple application of Nevai's theorem, as follows.

LEMMA 1. Let $k$ satisfy the condition in Theorem 1 , and let $f$ be continuous and satisfy (3.1). Then

$$
\lim _{n \rightarrow \infty} I_{n}(k, f)=I(k, f) .
$$

Proof. From the definitions we have

$$
\begin{aligned}
& \left|I(k, f)-I_{n}(k, f)\right|=\left|\int_{-\infty}^{\infty} k(x)\left[f(x)-L_{n}^{f}(x)\right] d x\right| \\
& \quad \leqslant\left[\int_{-\infty}^{\infty}\left|k(x) e^{x^{2} / 2}\right|^{p} d x\right]^{1 / p}\left[\int_{-\infty}^{\infty}\left|f(x)-L_{n}^{f}(x)\right|^{q} e^{-q x^{2} / 2} d x\right]^{1 / q},
\end{aligned}
$$

where we have again used the Hölder inequality, with $q=p /(p-1)>1$. The lemma now follows from Nevai's theorem.

To prove the rest of Theorem 1, Nevai's theorem needs to be used in a less trivial way. First, we need some notation. For $1<p<\infty$ let $\|\cdot\|_{p}^{(+)}$and $\|\cdot\|_{p}^{(-)}$denote the norms defined by

$$
\|u\|_{p}^{( \pm)}=\left[\int_{-\infty}^{\infty}\left|u(x) e^{ \pm x^{2} / 2}\right|^{p} d x\right]^{1 / p},
$$

and let $L_{p}^{( \pm)}$denote the corresponding Banach spaces. Further, let $C_{h, 0}$ denote the space of functions $f \in C(\mathbf{R})$ that also satisfy

$$
\lim _{|x| \rightarrow \infty} \frac{f(x)}{h(x)}=0
$$


where $h$ is given by (2.5), and define the norm $\|\cdot\|_{h}$ as

$$
\|f\|_{h}=\|f / h\|_{\infty} .
$$

Then $C_{h, 0}$ is a Banach space under this norm. With this notation Nevai's theorem states that for $1<q<\infty$ we have

$$
\lim _{n \rightarrow \infty}\left\|L_{n}^{f}-f\right\|_{q}^{(-)}=0
$$

for all $f$ in $C_{h, 0}$, from which it follows trivially that $\left\|L_{n}^{f}\right\|_{q}^{(-)} \rightarrow\|f\|_{q}^{(-)}<\infty$, and hence

$$
\sup _{n}\left\|L_{n}^{f}\right\|_{q}^{(-)}<\infty
$$

for all $f \in C_{h, 0}$.

The following lemma is the cornerstone of the remainder of the proof of Theorem 1 .

LeMma 2. If $1<p<\infty$, then for all $k \in L_{p}^{(+)}$

$$
\sum_{i=1}^{n}\left|w_{n i}(k)\right| h\left(x_{n i}\right) \leqslant C_{p}\|k\|_{p}^{(+)}
$$

where

$$
C_{p}=\sup _{n} \sup _{f \in C_{h, 0}} \frac{\left\|L_{n}^{f}\right\|_{q}^{(-)}}{\|f\|_{h}}<\infty
$$

with $1 / p+1 / q=1$.

Proof. It is easily verified that

$$
\begin{aligned}
\sum_{i=1}^{n}\left|w_{n i}(k)\right| h\left(x_{n i}\right) & =\sup _{f \in C_{h, 0}} \frac{\left|\sum_{i=1}^{n} w_{n i}(k) f\left(x_{n i}\right)\right|}{\|f\|_{h}}=\sup _{f \in C_{h, 0}} \frac{\left|\int_{-\infty}^{\infty} k(x) L_{n}^{f}(x) d x\right|}{\|f\|_{h}} \\
& \leqslant \sup _{f \in C_{h, 0}} \frac{\|k\|_{p}^{(+)}\left\|L_{n}^{f}\right\|_{q}^{(-)}}{\|f\|_{h}} \leqslant C_{p}\|k\|_{p}^{(+)},
\end{aligned}
$$

where $C_{p}$ is given by (3.5). That $C_{p}$ is a finite number follows from (3.4) by an application of the uniform boundedness theorem, because $C_{h, 0}$ is a Banach space.

Now suppose that $p>1$ is a fixed number for which the condition (2.1) is satisfied, and that $f$ satisfies the conditions stated in the theorem. We seek first to prove (2.4), the convergence property for the companion rule. The argument is analogous to one used in [21].

Our strategy will be to approximate the function $k$ by another function $k^{*}$, which satisfies (2.1), but which is constructed in a special way, to be described later. By 
elementary inequalities we then have

$$
\begin{aligned}
& \left|\sum_{i=1}^{n}\right| w_{n i}(k)\left|f\left(x_{n i}\right)-\int_{-\infty}^{\infty}\right| k(x)|f(x) d x| \\
& \leqslant \int_{-\infty}^{\infty}|| k^{*}(x)|-| k(x)|||f(x)| d x+\sum_{i=1}^{n}|| w_{n i}(k)|-| w_{n i}\left(k^{*}\right)||\left|f\left(x_{n i}\right)\right| \\
& \quad+\left|\sum_{i=1}^{n}\right| w_{n i}\left(k^{*}\right)\left|f\left(x_{n i}\right)-\int_{-\infty}^{\infty}\right| k^{*}(x)|f(x) d x| .
\end{aligned}
$$

Now

$$
|| w_{n i}(k)|-| w_{n i}\left(k^{*}\right)|| \leqslant\left|w_{n i}(k)-w_{n i}\left(k^{*}\right)\right|=\left|w_{n i}\left(k-k^{*}\right)\right|,
$$

since $w_{n i}(k)$ is linear in $k$; see Section 5. Moreover, by the assumption on $f$ in the theorem, the norm $\|f\|_{h}$ defined by (3.3) is finite, and we can write, for all $x \in \mathbf{R}$,

$$
|f(x)| \leqslant\|f\|_{h} h(x) \text {. }
$$

Hence, using Lemma 2, the second term on the right-hand side of (3.6) satisfies

$$
\begin{aligned}
\sum_{i=1}^{n}\left\|w_{n i}(k)|-| w_{n i}\left(k^{*}\right)\right\|\left|f\left(x_{n i}\right)\right| & \leqslant\|f\|_{h} \sum_{i=1}^{n}\left|w_{n i}\left(k-k^{*}\right)\right| h\left(x_{n i}\right) \\
& \leqslant\|f\|_{h} C_{p}\left\|k-k^{*}\right\|_{p}^{(+)} .
\end{aligned}
$$

The first term on the right-hand side of (3.6) satisfies

$$
\begin{aligned}
\int_{-\infty}^{\infty}\left\|k^{*}(x)|-| k(x)\right\||f(x)| d x & \leqslant\|f\|_{h} \int_{-\infty}^{\infty}\left|k^{*}(x)-k(x)\right| h(x) d x \\
& \leqslant\|f\|_{h}\|h\|_{q}^{(-)}\left\|k^{*}-k\right\|_{p}^{(+)}
\end{aligned}
$$

again using the Hölder inequality. Note that

$$
\|h\|_{q}^{(-)}=\left[\int_{-\infty}^{\infty}\left(1+x^{2}\right)^{-q / 2} d x\right]^{1 / q}<\infty,
$$

because $q>1$.

Thus the first two terms of (3.6) are bounded by constant multiples of $\left\|k^{*}-k\right\|_{p}^{(+)}$. Now we write

$$
k(x)=e^{-x^{2}} K(x), \quad k^{*}(x)=e^{-x^{2}} K^{*}(x),
$$

so that

$$
\left\|k^{*}-k\right\|_{p}^{(+)}=\left\|K^{*}-K\right\|_{p}^{(-)} .
$$

It follows from the assumption (2.1) that $K \in L_{p}^{(-)}$. Let us now require that $K^{*}$ be a polynomial. Since the polynomials are dense in the space $L_{p}^{(-)}$(see [13, Lemma 2]), we can choose $K^{*}$ so that the first two terms on the right-hand side of (3.6) are each less than $\varepsilon$, where $\varepsilon>0$ is a given arbitrary number.

It only remains to show that, having thus chosen $K^{*}$, the third term on the right-hand side of (3.6) is less than $\varepsilon$ for all $n$ sufficiently large. Since $K^{*}$ is a polynomial, of degree say $m$, it is shown in Section 5 (see (5.6)) that for $n>m$ we have

$$
w_{n i}\left(k^{*}\right)=\mu_{n i} K^{*}\left(x_{n i}\right)
$$


where $\mu_{n i}$ is the Gauss-Hermite quadrature weight that corresponds to the point $x_{n i}$; and hence

$$
\begin{aligned}
& \left|\sum_{i=1}^{n}\right| w_{n i}\left(k^{*}\right)\left|f\left(x_{n i}\right)-\int_{-\infty}^{\infty}\right| k^{*}(x)|f(x) d x| \\
& \quad=\left|\sum_{i=1}^{n} \mu_{n i}\right| K^{*}\left(x_{n i}\right)\left|f\left(x_{n i}\right)-\int_{-\infty}^{\infty} e^{-x^{2}}\right| K^{*}(x)|f(x) d x|,
\end{aligned}
$$

which is the error in the Gauss-Hermite quadrature rule for the function $\left|K^{*}(x)\right|$ $\times f(x)$. The latter function is Riemann integrable over each finite subinterval of $\mathbf{R}$ and satisfies

$$
\left|K^{*}(x) f(x)\right| \leqslant A \frac{e^{x^{2}}}{(1+|x|)^{2}},
$$

where $A$ is a constant, which is a sufficient condition for convergence of the Gauss-Hermite rule [24, p. 559]. Hence the last term on the right side of (3.6) can be made less than $\varepsilon$ by taking $n$ sufficiently large, and so the proof of the companion rule (2.4) is complete.

The convergence property (2.3), for the conditions stated in the theorem, can be proved in an almost identical fashion: all that is required is the omission of some of the absolute value signs from the argument used to prove (2.4).

4. Proof of Theorem 2 . Let $p_{n-1}$ be an arbitrary polynomial of degree $\leqslant n-1$. Then, because the rule $I_{n}(k, f)$ is exact if $f$ is such a polynomial, it follows that

$$
\begin{aligned}
& \left|I(k, f)-I_{n}(k, f)\right|=\left|I\left(k, f-p_{n-1}\right)-I_{n}\left(k, f-p_{n-1}\right)\right| \\
& \quad=\left|\int_{-\infty}^{\infty} k(x) g(x) \frac{f(x)-p_{n-1}(x)}{g(x)} d x-\sum_{i=1}^{n} w_{n i}(k) g\left(x_{n i}\right) \frac{f\left(x_{n i}\right)-p_{n-1}\left(x_{n i}\right)}{g\left(x_{n i}\right)}\right| \\
& \quad \leqslant\left[\int_{-\infty}^{\infty}|k(x)| g(x) d x+\sum_{i=1}^{n}\left|w_{n i}(k)\right| g\left(x_{n i}\right)\right]\left\|f-p_{n-1}\right\|_{g} .
\end{aligned}
$$

Because this holds for any polynomial of degree $\leqslant n-1$, the desired result follows on taking $p_{n-1}$ to be the polynomial of best approximation to $f$ in the sense of the norm $\|\cdot\|_{g}$.

5. Practical Construction of the Rules. The construction is a special case of that given by [5], [8], [15] for product integration based on the zeros of orthogonal polynomials.

Let $H_{n}$ be the Hermite polynomial of degree $n$ normalized by

$$
\int_{-\infty}^{\infty} e^{-x^{2}} H_{l}(x) H_{m}(x) d x=h_{l} \delta_{l m}=2^{l} l ! \pi^{1 / 2} \delta_{l m}
$$

and let $\left\{x_{n i}\right\}_{i=1}^{n}$ and $\left\{\mu_{n i}\right\}_{i=1}^{n}$ be the points and weights in the Gauss-Hermite quadrature formula

$$
\int_{-\infty}^{\infty} e^{-x^{2}} g(x) d x \approx \sum_{i=1}^{n} \mu_{n i} g\left(x_{n i}\right) .
$$


Then, because the Gauss-Hermite rule is exact if $g$ is a polynomial of degree $<2 n$, it follows that the Hermite polynomials also satisfy the discrete orthogonality relation

$$
\sum_{i=1}^{n} \mu_{n i} H_{l}\left(x_{n i}\right) H_{m}\left(x_{n i}\right)=h_{l} \delta_{l m},
$$

provided that $l+m<2 n$.

If $L_{n}^{f}$ is the unique polynomial of degree $<n$ that coincides with $f$ at $x_{n 1}, \ldots, x_{n n}$, then it follows from the discrete orthogonality relation that

$$
L_{n}^{f}(x)=\sum_{l=0}^{n-1} b_{n l} H_{l}(x)
$$

where

$$
b_{n l}=\frac{1}{h_{l}} \sum_{i=1}^{n} \mu_{n i} H_{l}\left(x_{n i}\right) f\left(x_{n i}\right) .
$$

It then follows from (1.2) that

$$
I_{n}(k, f)=\sum_{l=0}^{n-1} a_{l} b_{n l}
$$

where

$$
a_{l}=\int_{-\infty}^{\infty} k(x) H_{l}(x) d x, \quad l=0,1, \ldots,
$$

or alternatively

$$
I_{n}(k, f)=\sum_{i=1}^{n} w_{n i}(k) f\left(x_{n i}\right),
$$

where

$$
w_{n i}(k)=\mu_{n i} \sum_{l=0}^{n-1} \frac{a_{l}}{h_{l}} H_{l}\left(x_{n i}\right) .
$$

Note that $a_{l}$ may be written as

$$
a_{l}=\int_{-\infty}^{\infty} e^{-x^{2}} K(x) H_{l}(x) d x
$$

where $K$ is defined by (3.7). Hence we may write

$$
w_{n i}(k)=\mu_{n i} S_{n-1}^{K}\left(x_{n i}\right),
$$

where

$$
S_{n-1}^{K}(x)=\sum_{l=0}^{n-1} \frac{a_{l}}{h_{l}} H_{l}(x),
$$

the $n$th partial sum of the Fourier-Hermite series corresponding to the function $K$. In particular, if $K$ is a polynomial of degree $m$, then for $n>m$ we have $S_{n-1}^{K}(x)=$ $K(x)$, and hence for this special case

$$
w_{n i}(k)=\mu_{n i} K\left(x_{n i}\right) \text {. }
$$

(This property is used in the proof of Theorem 1.) 
In practice $I_{n}(k, f)$ may be evaluated by either (5.2) or (5.4), according to taste. In the calculations of Section 7 we have in fact used the latter, with the Clenshaw recurrence method [4] used to evaluate the sum in (5.5).

The amount of computation may be reduced in special cases by exploiting symmetries of $f$ or $k$. First, if $f$ is either even or odd, then one need calculate $f\left(x_{n i}\right)$ only for nonnegative points $x_{n i}$, because the points $x_{n i}$ are symmetric about the origin. Second, if $k$ is even, then $a_{l}=0$ for odd values of $l$, and the weights $w_{n i}(k)$, like the Gauss-Hermite weights $\mu_{n i}$, are equal for a symmetric pair of points. Finally, if $k$ is odd, then $a_{l}=0$ for even values of $l$, and the weights for a symmetric pair of points differ only in sign.

The heart of any practical product-integration calculation is the computation of the moments $a_{l}, l=0,1, \ldots$, for the given function $k$. We conclude this section with suggested computational schemes for some simple yet important choices of $k$. For these examples we use readily available analytical results. For other functions $k$ of practical interest, appropriate analytical techniques for calculating the moments are required.

$$
\begin{aligned}
& \text { A } k(x)=e^{-\alpha^{2} x^{2}} \cos \beta x, \quad \alpha>0, \\
& \text { B } k(x)=e^{-\alpha^{2} x^{2}} \sin \beta x, \quad \alpha>0 \text {. }
\end{aligned}
$$

In the first case $a_{l}$ vanishes if $l$ is odd, and in the second $a_{l}$ vanishes if $l$ is even. The remaining values of $a_{l}$ are given in both cases by [10, p. 840]

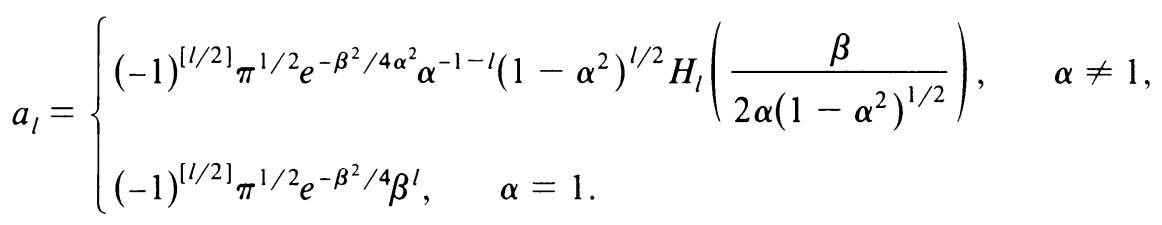

(Note that the argument of the Hermite polynomial is imaginary if $\alpha>1$. In that situation either branch of $\left(1-\alpha^{2}\right)^{1 / 2}$ may be taken.)

$$
\begin{array}{lll}
\text { C } & k(x)=e^{-\alpha^{2} x^{2}} \cos \beta^{2} x^{2}, & \alpha>0, \\
\text { D } & k(x)=e^{-\alpha^{2} x^{2}} \sin \beta^{2} x^{2}, & \alpha>0 .
\end{array}
$$

In both cases $a_{l}=0$ if $l$ is odd. If $l$ is even, then by [10, p. 839] $a_{l}$ is the real or imaginary part, respectively, of

$$
A_{l}=\pi^{1 / 2} \frac{l !}{(l / 2) !}\left(1-\alpha^{2}+i \beta^{2}\right)^{l / 2}\left(\alpha^{2}-i \beta^{2}\right)^{-l / 2-1 / 2} .
$$

The complex numbers $A_{l}$, or their real and imaginary parts separately, can easily be generated by recurrence on $l$.

$$
\begin{array}{ll}
\text { E } k(x)=|x| e^{-\alpha^{2} x^{2}} \cos \beta^{2} x^{2}, & \alpha>0, \\
\text { F } k(x)=|x| e^{-\alpha^{2} x^{2}} \sin \beta^{2} x^{2}, & \alpha>0 .
\end{array}
$$

Again $a_{l}=0$ if $l$ is odd. If $l$ is even, then $a_{l}$ is the real or imaginary part, respectively, of

$$
A_{l}=2^{l}\left(\frac{l}{2}\right) ! \sum_{n=0}^{l / 2} \frac{\left(\frac{1}{2}-\frac{l}{2}\right)\left(\frac{3}{2}-\frac{l}{2}\right) \cdots\left(n-\frac{1}{2}-\frac{l}{2}\right)}{n !}\left(\alpha^{2}-i \beta^{2}\right)^{n-l / 2-1}
$$


This is obtained by substituting into (5.3) the explicit expression for the polynomial $H_{l}(x)$ (see [1, p. 775]).

$$
\text { G } k(x)=e^{-\alpha^{2} x^{2} \theta(x-a) \theta(b-x), \quad \alpha \geqslant 0 .}
$$

Here $-\infty<a<b<+\infty$, and $\theta(x)$ is the step function

$$
\theta(x)= \begin{cases}0 & \text { if } x<0 \\ 1 & \text { if } x \geqslant 0\end{cases}
$$

If $\alpha>0$, we may also allow $a=-\infty$ or $b=+\infty$, since the integral for $a_{l}$,

$$
a_{l}=\int_{a}^{b} e^{-\alpha^{2} x^{2}} H_{l}(x) d x
$$

remains well defined.

If $\alpha=0$, then by using the relation

$$
H_{l}(x)=\frac{1}{2(l+1)} H_{l+1}^{\prime}(x), \quad l \geqslant 0,
$$

we obtain

$$
a_{l}=\frac{1}{2(l+1)}\left[H_{l+1}(b)-H_{l+1}(a)\right]
$$

On the other hand if $\alpha>0$ and $-\infty<a<b<\infty$, then since $H_{0}(x)=1$ we obtain

$$
a_{0}=\frac{\pi^{1 / 2}}{2 \alpha}[\operatorname{erf}(b \alpha)-\operatorname{erf}(a \alpha)]
$$

where $\operatorname{erf}(t)$ is the error function. The remaining moments $a_{l}, l \geqslant 1$, may then be obtained by upward recurrence, as follows. First, for $l \geqslant 2$ we substitute the relation

$$
H_{l}(x)=2 x H_{l-1}(x)-(2 l-2) H_{l-2}(x), \quad l \geqslant 2,
$$

into (5.7) and then integrate by parts in the first term and use (5.8) to obtain the recurrence

$$
\begin{aligned}
a_{l}= & \frac{1}{\alpha^{2}}\left[e^{-\alpha^{2} a^{2}} H_{l-1}(a)-e^{-\alpha^{2} b^{2}} H_{l-1}(b)\right] \\
& +(2 l-2)\left(\frac{1}{\alpha^{2}}-1\right) a_{l-2}, \quad l \geqslant 2 .
\end{aligned}
$$

It can be verified easily that this relation holds also for $l=1$ if $a_{-1}$ is taken to be zero. Finally, the results for $a=-\infty$ or $b=+\infty$ follow from the above results by taking the appropriate limits.

Remark. The point of example $\mathrm{G}$ is that it allows us to evaluate

$$
\int_{a}^{b} e^{-\alpha^{2} x^{2}} f(x) d x
$$

for arbitrary values of $a$ and $b$, by a quadrature rule based on the one fixed set of points. For example, we may, if we wish, evaluate the integral from $-\infty$ to a variable upper limit $b$, i.e. find the indefinite integral. Of prime importance is the fact that for $\alpha^{2}>\frac{1}{2}$ the convergence of the quadrature rule approximating (5.11) is uniform with respect to $a$ and $b$, provided $f$ is continuous and satisfies (2.2). This follows from the 
error bound (3.2), which gives

$$
\begin{aligned}
\left|I(k, f)-I_{n}(k, f)\right| & \leqslant\left[\int_{a}^{b} e^{-p\left(\alpha^{2}-1 / 2\right) x^{2}} d x\right]^{1 / p}\left[\int_{-\infty}^{\infty}\left|f(x)-L_{n}^{f}(x)\right|^{q} e^{-q x^{2} / 2} d x\right]^{1 / q} \\
& \leqslant\left[\int_{-\infty}^{\infty} e^{-p\left(\alpha^{2}-1 / 2\right) x^{2}} d x\right]^{1 / p}\left[\int_{-\infty}^{\infty}\left|f(x)-L_{n}^{f}(x)\right|^{q} e^{-q x^{2} / 2} d x\right]^{1 / q}
\end{aligned}
$$

the right-hand side of which converges to zero as $n \rightarrow \infty$ by virtue of Nevai's mean-convergence theorem (see Section 3) for any fixed $q>1$.

6. Semi-Infinite Intervals. Consider the integral

$$
I_{+}(K, F)=\int_{0}^{\infty} K(t) F(t) d t
$$

where $K$ is integrable and $F$ is smooth. If $F$ has a smooth even extension to the whole real line (e.g., $F(t)=\cos t$ or $\left.F(t)=\left(1+t^{2}\right)^{-1}\right)$, then we may write

$$
I_{+}(K, F)=\frac{1}{2} \int_{-\infty}^{\infty} K(|x|) F(x) d x,
$$

which is in the form (1.1) required for the product-integration method if we take $k(x)=\frac{1}{2} K(|x|)$ and $f(x)=F(x)$. Similarly, if $F$ has an odd smooth extension to the whole real line (e.g., $F(t)=\sin t$ ), then we may write

$$
I_{+}(K, F)=\frac{1}{2} \int_{-\infty}^{\infty} \operatorname{sign}(x) K(|x|) F(x) d x,
$$

which is again of the required form if we take $k(x)=\frac{1}{2} \operatorname{sign}(x) K(|x|)$. In practice one or other of the above situations can often be achieved by a judicious manipulation of factors in the integrand.

In all other cases we may transform the variable in (6.1) by substituting $t=x^{2}$, to give

$$
I_{+}(K, F)=2 \int_{0}^{\infty} x K\left(x^{2}\right) F\left(x^{2}\right) d x=\int_{-\infty}^{\infty}|x| K\left(x^{2}\right) F\left(x^{2}\right) d x
$$

which is of the form required for the product integration method if we take, for example, $k(x)=|x| K\left(x^{2}\right)$ and $f(x)=F\left(x^{2}\right)$. An example of the use of this transformation is given in the following section.

Because of the correspondence between Hermite polynomials $H_{m}(x)$ and the Laguerre polynomials $L_{n}^{(1 / 2)}\left(x^{2}\right), L_{n}^{(-1 / 2)}\left(x^{2}\right)$ [23, Eq. (5.6.1)], the use of the present rule on the transformed integral can alternatively be interpreted as a product rule for $[0, \infty)$ using zeros of Laguerre polynomials. We do not detail this approach further in the present paper except to write down the corresponding form of Theorem 1.

THEOREM 1a. Let $K$ satisfy

$$
\int_{0}^{\infty}\left|K(t) e^{t / 2} t^{1 / 2}\right|^{p} t^{-1 / 2} d t<\infty
$$

for some $p>1$, and let $F$ be a function on $[0, \infty)$ which is Riemann integrable on every finite interval and which satisfies

$$
|F(t)| \leqslant C \frac{e^{t / 2}}{(1+t)^{1 / 2}}
$$


for some constant $C$. Then $K F \in L_{1}[0, \infty)$, and

$$
\lim _{n \rightarrow \infty} \sum_{i=1}^{n} W_{n i}(K) F\left(t_{n i}\right)=\int_{0}^{\infty} K(t) F(t) d t
$$

and

$$
\lim _{n \rightarrow \infty} \sum_{i=1}^{n}\left|W_{n i}(K)\right| F\left(t_{n i}\right)=\int_{0}^{\infty}|K(t)| F(t) d t
$$

if the nodes $\left\{t_{n i}\right\}$ for interpolatory product integration on $[0, \infty)$ are chosen either as

(a) the zeros of $L_{n}^{(-1 / 2)}(t)$, or

(b) the zeros of $L_{n-1}^{(1 / 2)}(t)$ supplemented by $t=0$, and $\left\{W_{n i}\right\}$ are the corresponding weights.

7. Numerical Examples. Numerical results for a number of examples are shown in Table 1. In each case other than (5b) analytical results were available to estimate numerical values for the integrals. (For (5b) the difference from (5a) was estimated by a many-point Simpson's rule.) The Gauss-Hermite weights $\left\{\mu_{n i}\right\}$ and nodes $\left\{x_{n i}\right\}$ were calculated using an algorithm of Golub and Welsch [11], and we are grateful to these authors for supplying a copy of their program. To guard against roundoff errors the calculations were implemented in double precision FORTRAN on the Cyber 171 at the University of New South Wales (29 significant decimal digits).

Example 1.

$$
\int_{-\infty}^{\infty} e^{-x^{2}+x} \sin 5 x d x
$$

Here we have chosen

$$
k(x)=e^{-x^{2}} \sin 5 x, \quad f(x)=e^{x} .
$$

Clearly, the rate of convergence is excellent, even though $f(x)$ increases exponentially at $\infty$. The ratio $\Sigma\left|w_{n i}\right| f\left(x_{n i}\right) / \int|k| f$, shown in the final column, appears to be converging to 1 , in conformity with Theorem 1 .

Example 2.

$$
\int_{-\infty}^{\infty} e^{-x^{2}} \cos 100 x^{2}\left(\frac{4-x^{2}}{4+x^{2}}\right) d x
$$

The integrand in this case is highly oscillatory. We take

$$
k(x)=e^{-x^{2}} \cos 100 x^{2}, \quad f(x)=\frac{4-x^{2}}{4+x^{2}},
$$

so that the oscillatory behavior is accounted for by $k$. Evidently, the rate of convergence is much slower than in Example 1, but is still very satisfactory. The ratios shown in the last column are rather smaller than the limit value 1, indicating good numerical stability against errors in the values of $f(x)$.

Example 3.

$$
\int_{0}^{\infty} e^{-t^{2}} \cos 100 t^{2}\left(\frac{1-t^{2}}{1+t^{2}}\right) d t=\frac{1}{2} \int_{-\infty}^{\infty} e^{-x^{2}} \cos 100 x^{2}\left(\frac{1-x^{2}}{1+x^{2}}\right) d x
$$

Again the integrand is highly oscillatory, and we take $k(x)=e^{-x^{2}} \cos 100 x^{2}$, as in Example 2, but now $f(x)=\frac{1}{2}\left(1+x^{2}\right) /\left(1-x^{2}\right)$. In each case $f$ is a simple rational 
TABLE 1. Numerical examples

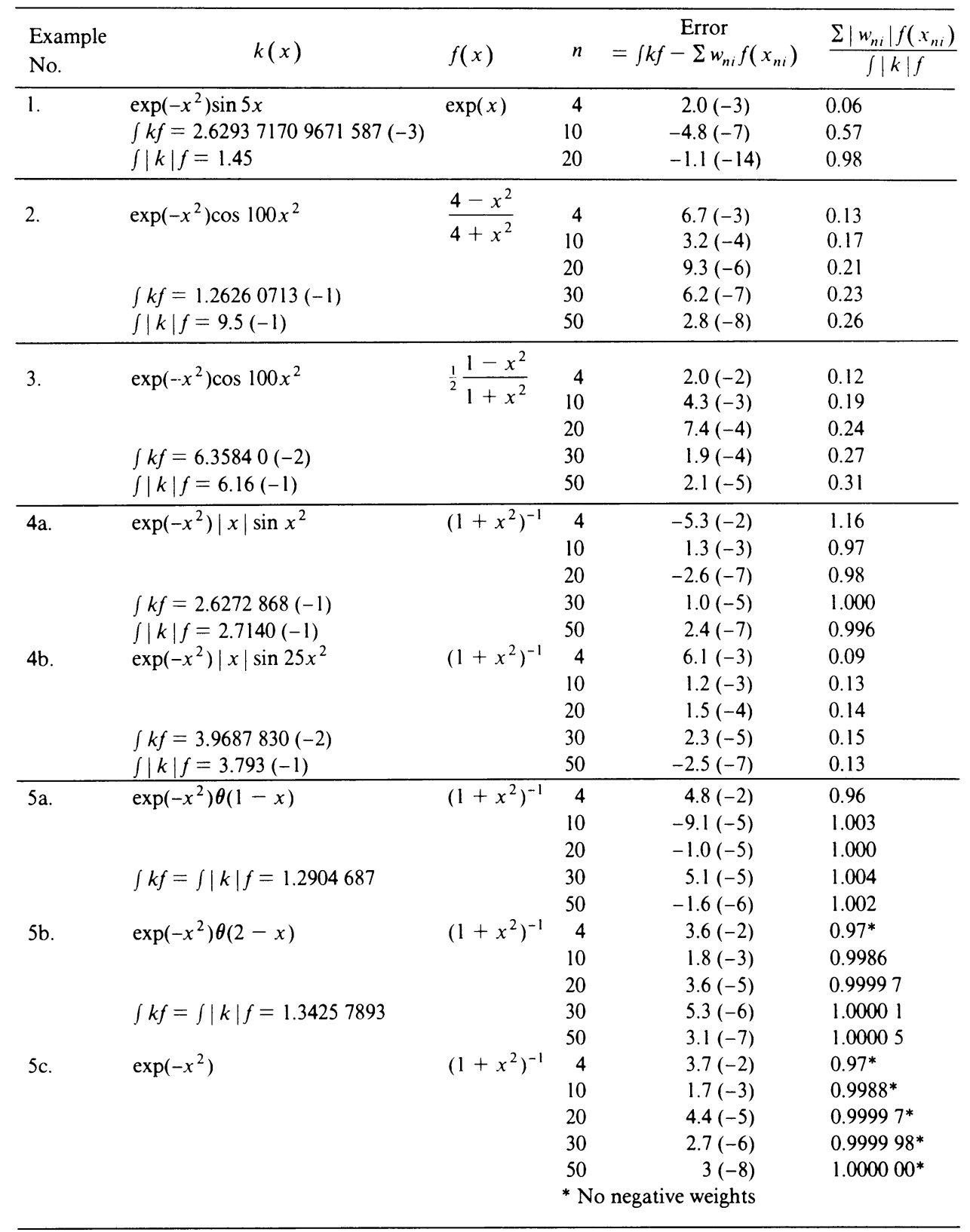

function, but in this example $f$ has poles at $\pm i$ compared with $\pm 2 i$ in Example 2 (i.e. nearer to the real axis), and the convergence is less rapid.

Example 4.

$$
\int_{0}^{\infty} e^{-t} \sin \gamma t \frac{1}{1+t} d t
$$

This is a semi-infinite integral which we may treat by applying the transformation $t=x^{2}$, as discussed in Section 6, to obtain

$$
\int_{-\infty}^{\infty} e^{-x^{2}} \sin \gamma x^{2} \frac{1}{1+x^{2}}|x| d x
$$


Hence we take

$$
k(x)=|x| e^{-x^{2}} \sin \gamma x^{2}, \quad f(x)=\frac{1}{1+x^{2}} .
$$

Numerical results are shown for $\gamma=1$ and $\gamma=25$. Note that the absolute errors in the two cases are comparable, as we might expect from the error bound of Corollary 1 .

\section{Example 5.}

$$
\int_{-\infty}^{b} e^{-x^{2}} \frac{1}{1+x^{2}} d x
$$

If $b$ is arbitrary, then the integral is the indefinite integral of $e^{-x^{2}} /\left(1+x^{2}\right)$. We take

$$
k(x)=e^{-x^{2} \theta} \theta(b-x), \quad f(x)=\frac{1}{1+x^{2}},
$$

where $\theta(x)$ is the unit step function. Numerical results are shown in the table for $b=1,2$ and $\infty$. Note that the apparent rate of convergence is broadly similar in the three cases, in conformity with the theoretical result proved in Section 5 that the convergence is uniform with respect to $b$. (Note that the case $b=\infty$ corresponds to Gauss-Hermite quadrature with $f(x)=1 /\left(1+x^{2}\right)$.)

Department of Applied Mathematics

University of New South Wales

Sydney, N.S.W. 2033, Australia

1. M. Abramowitz \& I. A. Stegun, Handbook of Mathematical Functions with Formulas, Graphs, and Mathematical Tables, Nat. Bur. Standards, Appl. Math. Series, No. 55, U. S. Government Printing Office, Washington, D. C., 1964.

2. N. S. Bakhvalov \& L. G. Vasil'Eva, "Evaluation of the integrals of oscillating functions by interpolation at nodes of Gaussian quadratures," U.S.S.R. Comput. Math. and Math. Phys., v. 8, 1968, pp. 241-249.

3. M. Blakemore, G. A. Evans \& J. Hyslop, "Comparison of some methods for evaluating infinite range oscillatory integrals," J. Comput. Phys., v. 22, 1976, pp. 352-376.

4. C. W. Clenshaw, "A note on the summation of Chebyshev series," MTAC, v. 9, 1955, pp. $118-120$.

5. D. Elliott \& D. F. Paget, "Product-integration rules and their convergence," BIT, v. 16, 1976, pp. $32-40$.

6. D. Elliott \& D. F. PAget, “The convergence of product integration rules," BIT, v. 18, 1978, pp. 137-141.

7. G. FreUd, "A contribution to the problem of weighted polynomial approximation," Linear Operators and Approximation (P. L. Butzer, J.-P. Kahane and B. Sz.-Nagy, Eds.), Birkhäuser Verlag, Berlin, 1971, pp. 431-447.

8. G. Freud, Orthogonal Polynomials, Pergamon Press, Oxford, 1971, Problem 1, pp. 130-131.

9. B. GabutTI, "On high precision methods for computing integrals involving Bessel functions," Math. Comp., v. 33, 1979, pp. 1049-1057.

10. I. S. Gradshteyn \& I. M. Ryzhik, Table of Integrals Series and Products, Academic Press, New York, 1965.

11. G. H. Golub \& J. H. Welsch, “Calculation of Gaussian quadrature rules," Math. Comp., v. 23, 1969. pp. 221-230.

12. D. R. Lehman, W. C. Parke \& L. C. Maximon, “Numerical evaluation of integrals containing a spherical Bessel function by product integration,” J. Math. Phys., v. 22, 1981, pp. 1399-1413.

13. B. Muckenhoupt, "Mean convergence of Hermite and Laguerre series. II," Trans. Amer. Math. Soc., v. 147, 1970, pp. 433-460.

14. P. G. NeVAI, “Mean convergence of Lagrange interpolation. II," J. Approx. Theory, v. 30, 1980, pp. 263-276.

15. D. F. Paget, Generalised Product Integration, Ph.D. thesis, University of Tasmania, 1976. 
16. T. N. L. Patterson, “On high precision methods for the evaluation of Fourier integrals with finite and infinite limits," Numer. Math., v. 27, 1976, pp. 41-52.

17. I. H. SLOAN, “On the numerical evaluation of singular integrals," BIT, v. 18, 1978, pp. 91-102.

18. I. H. SloAN, "On choosing the points in product integration," J. Math. Phys., v. 21, 1980, pp. 1032-1039.

19. I. H. Sloan \& W. E. Smith, "Product integration with the Clenshaw-Curtis and related points. Convergence properties," Numer. Math., v. 30, 1978, pp. 415-428.

20. I. H. SloAN \& W. E. Smith, "Product integration with the Clenshaw-Curtis points: Implementation and error estimates," Numer. Math., v. 34, 1980, pp. 387-401.

21. I. H. SLOAN \& W. E. Smith, "Properties of interpolatory product integration rules," SIAM J. Numer. Anal., v. 19, 1982, pp. 427-442.

22. W. E. Smith \& I. H. SloAN, "Product-integration rules based on the zeros of Jacobi polynomials," SIAM J. Numer. Anal., v. 17, 1980, pp. 1-13.

23. G. Szegö, Orthogonal Polynomials, Amer. Math. Soc. Colloq. Publ., Vol. 23, Amer. Math. Soc., Providence, R. I., 1939.

24. J. V. USPENSKY, "On the convergence of quadrature formulas related to an infinite interval," Trans. Amer. Math. Soc., v. 30, 1928, pp. 542-559.

25. A. Young, “Approximate product integration," Proc. Roy. Soc. London Ser. A, v. 224, 1954, pp. $552-561$. 